Neurosurg Focus 11 (4):Introduction, 2001, Click here to return to Table of Contents

\title{
CHANGING MANAGEMENT STRATEGIES FOR POSTTRAUMATIC INTRACRANIAL MASS LESIONS
}

\author{
Neurosurgical Focus, October 2001 \\ Volume 11, Issue 4 \\ Topic Editor: Alex B. Valadka, M.D. \\ Department of Neurosurgery, Baylor College of Medicine, Houston, Texas
}

In this issue of Neurosurgical Focus we draw together several recently published articles from the Journal of $\mathrm{Neu}$ rosurgery, and one new article in which the current evaluation and treatment of head injury is the primary topic.

The paper by Medele, et al., suggests that Terson syndrome may occur as commonly after severe brain injury as it does after aneurysmal subarachnoid hemorrhage, a fact that may be helpful for neurosurgeons to remember when more sophisticated diagnostic tools are unavailable.

In terms of monitoring patients who have sustained a traumatic brain injury (TBI), Cormio's paper reminds us that, even though low jugular venous oxygen saturation $\left(\mathrm{SjvO}_{2}\right)$ values are strongly associated with poor outcomes, one cannot assume the opposite-that is, that elevated $\mathrm{SjvO}_{2}$ values correlate with good outcomes. One implication of this work is that the usefulness of monitoring procedures is heavily dependent on the knowledge and judgment of those who use them. Questions raised by such reports may be more amenable to investigation because of work such as that discussed by Engelborghs, et al., who describe an experimental model that mimics several important clinical features of TBI.

In terms of treatment, the authors of a well-conducted multicenter trial published earlier this year, failed to demonstrate any benefit of applying moderate hypothermia in patients who have sustained a TBI. The authors of laboratory investigations, however, continue to report beneficial effects of hypothermia. In the paper by Zhao, et al., the authors demonstrate that the mechanisms underlying such benefit are far from clear. Unraveling such mysteries may lead to the ability to identify particular patients in whom hypothermia is most likely to be beneficial. Regarding manipulation of cerebral perfusion pressure (CPP), the finding of Juul, et al., reinforces work by others, underscoring that the widely disseminated but data-deficient practice of elevating CPP to $70 \mathrm{~mm} \mathrm{Hg}$ in patients with severe TBI remains unproven. These authors suggest that there appears to be little additional benefit of increasing $\mathrm{CPP}$ as long as it remains above $60 \mathrm{~mm} \mathrm{Hg}$. More importantly, they emphasize the importance of treating elevations in intracranial pressure (ICP) above $20 \mathrm{~mm} \mathrm{Hg}$. Along the same line of validating established therapies, as opposed to eagerly embracing newer unproven ones, Kerr and colleagues report an investigation on how draining different amounts of cerebrospinal fluid affects ICP.

It is an honor to have been given the opportunity to comment briefly on these papers. Hopefully, the overview provided by these articles will inspire others to investigate some of the many remaining questions about optimal evaluation and treatment of head-injured patients. 